\section{Nonvolatile Programmable Spin- Logic Gates Show Potential in Reconfigurable Computing}

Researchers at Siemens AG and the University of Bielefeld, Germany, have made programmable spin-logic gates based on spin-dependent tunneling (STD) elements. The SDT elements form the spin-logic gates, allowing programmable logic operations.

For reconfigurable computing using highly magnetoresistive thin-film magnetic structures, it is essential that a programming operation be performed rapidly and with no practical limitation of programming cycles. In spin logic, the programming information (i.e., the logic inputs and outputs) is nonvolatile and power consumption is low. The researchers chose a tunneling system because it exhibits good readout-voltage levels for submicrometer elements and compatibility to nonvolatile memory. The major challenges of such a tunneling system are that it needs a homogeneous device-fabrication process and homogeneous tunneling-current distribution or a tunneling barrier. The researchers showed that such requirements could be met down to an area of 0.6 $\mu \mathrm{m}^{2}$ per SDT element.

Using a seven-mask lithography process, R. Richter and colleagues fabricated an $\mathrm{IrMn} / \mathrm{CoFe} / \mathrm{Ru} / \mathrm{CoFe} / \mathrm{Al}_{2} \mathrm{O}_{3} /$ $\mathrm{NiFe}$ tunneling structure, in which the artificial antiferrimagnet hard subsystem, $\mathrm{CoFe}(2.0 \mathrm{~nm}) / \mathrm{Ru}(0.9 \mathrm{~nm}) / \mathrm{CoFe}(3.0 \mathrm{~nm})$, was exchange-biased to the antiferromagnet IrMn. As reported in the February 18 issue of Applied Physics Letters, field-programmable gate arrays (FPGAs) were constructed from three-input fieldprogrammable spin-logic gates (in order to compare with semiconductor-based FPGAs). Each gate consisted of six SDT elements. A current in a switch line generated a magnetic field to switch the soft magnetic NiFe layer and, hence, the corresponding SDT element between its highand low-resistance states. A clock line ran on top and perpendicular to the switch line. These two lines were electrically isolated from each other and from the SDT elements. The device was typically operated at a tunneling current of $5 \mu \mathrm{A}$ with a corresponding voltage of $\sim 300 \mathrm{mV}$ across each SDT element. The voltage across a switch line led to a current and, hence, to a magnetic field at the location of the selected SDT element.

In the six-element configuration, three elements were chosen as reference bits, and the other three served as logic input bits. The voltages defining the inputs Boolean 0 and Boolean 1 were chosen such that the corresponding magnetic fields were sufficient to switch the soft magnetic layer of any selected SDT element. Thus, programming was as fast as performing a logic operation. Additionally, the researchers reported no limitation of programming cycles.

The researchers showed the feasibility of a hybrid field-programmable spinlogic gate based on SDT elements in steady-state and clocked operation. They achieved working hybrid spin-logic gates with higher integration density and the possibility of more than three inputs. The programming information and the logic inputs/output were nonvolatile. The researchers said that FPGAs based on spin-logic gates hold promise for reconfigurable computing.

SHIMING WU

\section{Light-Induced Transformation Changes Gallium Conductivity}

A research group led by Nikolay Zheludev at the University of Southampton has observed a new mechanism of photoconductivity in $\alpha$-gallium wherein a phase transition induced by light leads to a metastable phase of different conductivity. As reported in the February 18 issue of Applied Physics Letters, the researchers believe that the light-induced transformation was made possible by surface confinement of the gallium, which may have erased the distinction between first- and second-order phase transitions.

The researchers created the confined interface by sandwiching a small drop of liquid gallium between a cover glass and a glass substrate with silver electrodes spaced $250 \mu \mathrm{m}$ apart. The drop of liquid gallium was placed between the electrodes. Pressure on the cover glass flattened the drop so that it spanned the electrodes. Once solidified, the gallium "bridge" across the electrode gap was $\sim 50 \mu \mathrm{m}$ wide and $1 \mu \mathrm{m}$ thick. A 514-nm argon laser induced the conductivity change. The researchers focused the beam on a $30-\mu \mathrm{m}$ spot and mechanically modulated its intensity at $200 \mathrm{~Hz}$. In addition, a small dc current source and a lock-in detector were used to measure the change in conductivity. The researchers controlled the gallium temperature by using a Peltier heat pump in thermal contact with the glass substrate on which the silver electrodes were deposited.

While increasing the laser power, the researchers held the temperature at a constant $20^{\circ} \mathrm{C}$, which is below gallium's melting point. The light-induced conductivity change increased linearly with increasing power below $4 \mathrm{~mW}$, and then increased rapidly with increasing power from about $4 \mathrm{~mW}$ to $7.5 \mathrm{~mW}$, where the conductivity saturated. The researchers also used constant laser power while scanning the temperature across gallium's bulk melting point $\left(29.8^{\circ} \mathrm{C}\right)$. The resulting data showed a hysteresis in the photoconductivity signal with temperature. The researchers concluded that their sample underwent "surface-assisted light-induced metallization of $\alpha$-gallium." The researchers said that this material property has the potential for applications in optically fast broadband photodetectors.

PAMELA JOHNSON

\section{Laser-Induced Damage on CVD Diamond Measured by Photoacoustic Technique}

Researchers in the Department of Electrical Engineering at the National Tsinghua University, Taiwan, and at Brookhaven National Laboratory have measured the laser-induced damage threshold (LIDT) at the surface of bulk chemical-vapor-deposited (CVD) diamond. The LIDT was measured by amplifying the acoustic wave generated by the breakdown of the substrate under laser irradiation. The researchers said that this method enables a more accurate determination of substrate breakdown than other techniques currently employed in the field. The researchers found that the LIDT for the diamond surface was higher than that for other common $\mathrm{CO}_{2}$ laser optical materials under the same conditions. The exact improvement depended on the length of the laser pulse. These results, they said, indicate that CVD diamond is a promising material for $\mathrm{CO}_{2}$ laser optics.

As reported in the February 1 issue of Optics Letters, lead author A.C. Chiang and colleagues used CVD diamond, $\mathrm{ZnSe}$, and Ge window flats polished to an optical finish. The laser pulses were generated by a hybrid transversely excited atmosphere single longitudinal mode $\mathrm{CO}_{2}$ oscillator and amplifier. The oscillator generated 100-ns pulses; 200-ps pulses were generated by reflection of a portion of the 100-ns pulse from an optically gated Ge switch. LIDT is defined as the minimum laser fluence (energy per unit area) needed to damage the surface of the substrate. The LIDT for irradiation with 200-ps pulses was measured by placing the substrate in the path of the laser beam and then varying the attenuation of the beam until laserinduced dielectric breakdown was detected. The LIDT for irradiation with 100-ns pulses was measured by slowly moving the substrate toward the waist (the thinnest part) of an unattenuated laser beam until damage was detected. The error in the LIDT was determined from the variation in the laser power under the con- 
ditions used to induce breakdown.

The LIDT for the diamond substrate was determined to be $1.20 \mathrm{~J} / \mathrm{cm}^{2}$ and 8.0 $\mathrm{J} / \mathrm{cm}^{2}$ for $200-\mathrm{ps}$ and $100-\mathrm{ns}$ pulse irradiation, respectively. The LIDT for diamond was found to be three times higher than for $\mathrm{ZnSe}$, and five to six times higher than for $\mathrm{Ge}$, depending on pulse length.

GREG KHITROV

\section{Ultraslow and Stored Light Observed in Crystalline Solid}

Ultraslow and stored light have been observed in a Pr-doped $\mathrm{Y}_{2} \mathrm{SiO}_{5}$ crystalline solid. A. Turukhin of JDS Uniphase and colleagues affiliated with the Massachusetts Institute of Technology, Texas A\&M University, the Electronics and Telecommunications Research Institute in South Korea, and the Air Force Research Laboratory at Hanscom Air Force Base demonstrated light speeds as slow as $45 \mathrm{~m} / \mathrm{s}$ and even light-pulse "stopping."

Slow-light experiments work by a dramatic reduction in group velocity. To significantly slow or "stop" light, it is desirable for a material to possess a sharp feature in its dispersion relation. Such featuresunusual in solid materials-can be achieved in certain insulators doped with rare-earth elements. The researchers in this study used Pr-doped $\mathrm{Y}_{2} \mathrm{SiO}_{5}$, which has previously been shown to exhibit electromagnetically induced transparency (EIT), and which has also been a crucial feature in atomic gases previously used for demonstrating ultraslow light.

As reported in the January 14 issue of Physical Review Letters, the crystal was optically pumped by coupling and probe laser fields and produced a "spectral hole," a region where the crystal is transparent to the laser field. Simultaneously, an auxiliary field was required to produce an absorbing "antihole." This auxiliary field not only controlled the probe field absorption, but also reduced the effective inhomogeneous linewidth the coupling field must overcome to achieve EIT. The group light velocity was measured by chopping the probe and determining the phase delay of the chopped signal.

Individual probe pulse slowing could also be measured. An advantage of this EIT-based technique was that the group delays depended mostly on the intensity of the coupling laser. This meant that the group light speed could be altered by changing the coupling laser intensity and that complex probe pulse shapes could be effectively slowed with bandwidth serving as the only real limitation. Finally, the researchers demonstrated "stopped light." This phenomenon occurs when the group velocity is decreased to zero while also reducing the coupling laser intensity to

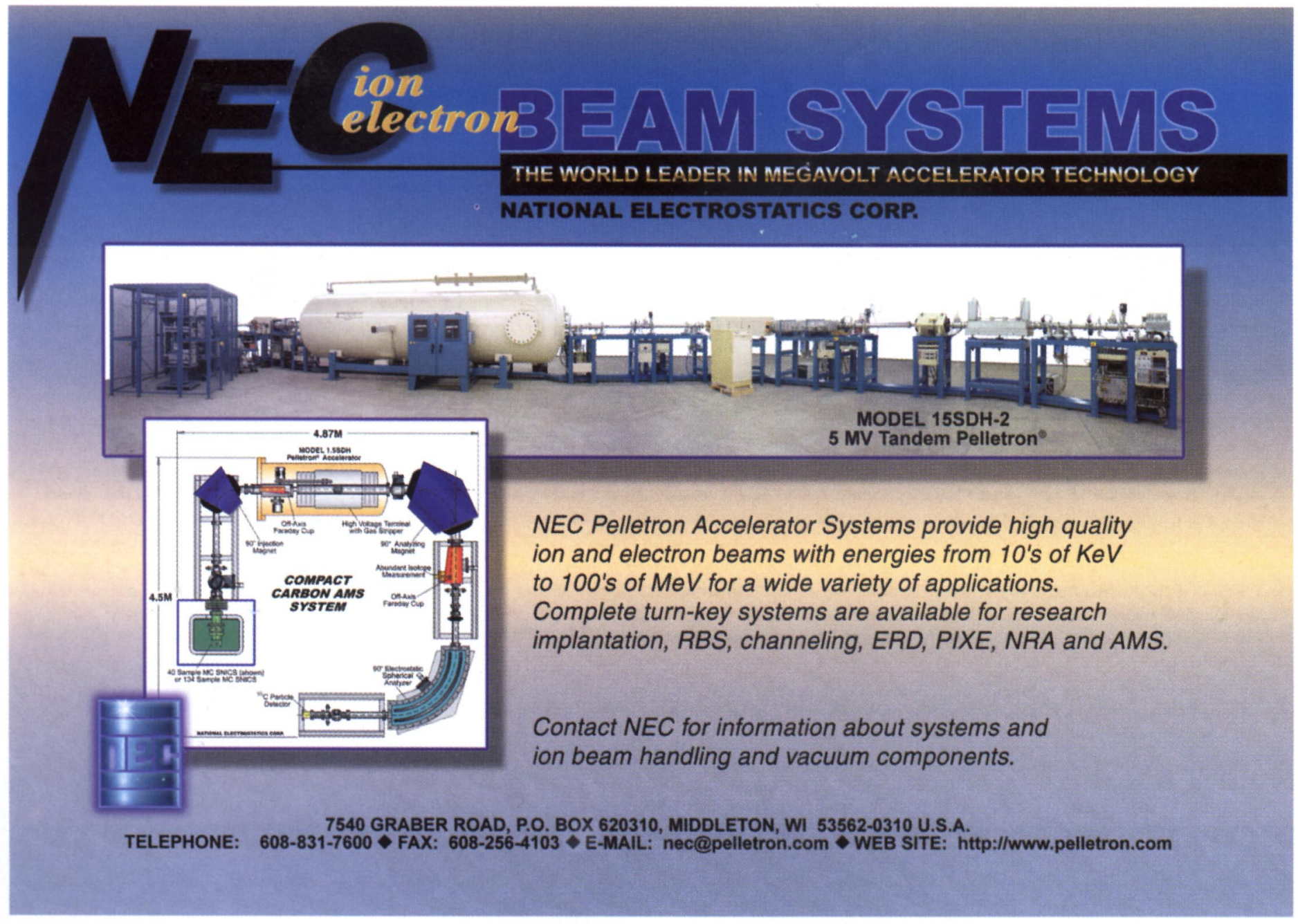

